\title{
Multiplex PCR for cryptococcal meningoencephalitis - calling for caution!!!
}

\begin{abstract}
An early diagnosis of cryptococcal meningoencephalitis is needed for prevention of significant morbidity and mortality. We present two cases of culture positive cryptococcal meningoencephalitis where the cerebrospinal fluid (CSF) molecular PCR panel failed to detect the Cryptoccoccus resulting in delay in diagnosis. Careful interpretation of such molecular platforms and multiple testing methods including CSF cryptococcal antigen $(\mathrm{CrAG})$ and cultures are advised in suspected cryptococcal meningitis.
\end{abstract}

Keywords: cryptococcal meningitis, bio fire filmarray meningitis/encephalitis panel, molecular diagnosis, cryptococcal meningoencephalitis, cryptococcal Ag test
Volume 8 Issue 2 - 2020

\author{
Neha Gupta,' Ravi Bhushan,' Atmaram \\ Bansal, ${ }^{2}$ Arun Garg, ${ }^{2}$ Jayesh Modi, ${ }^{3}$ Rajiv \\ Gupta, ${ }^{3}$ Reeshika Verma, ${ }^{3}$ Dinesh Bansal, ${ }^{4}$ \\ Vijay Kher, ${ }^{4}$ Rashmi, ${ }^{5}$ Teena Wadhwa, ${ }^{5}$ Smita \\ Sharma, ${ }^{5}$ Rajeev Soman ${ }^{6}$ \\ 'Division of Infectious Diseases, Medanta- The Medicity, India \\ ${ }^{2}$ Department of Neurology, Medanta- The Medicity, India \\ ${ }^{3}$ Department of Radiology, Medanta-The Medicity, India \\ ${ }^{4}$ Institute of Nephrology, Medanta- The Medicity, India \\ ${ }^{5}$ Department of Microbiology, Medanta- The Medicity, India \\ ${ }^{6}$ Consultant Infectious Diseases, Jupiter Hospital, India
}

Correspondence: Dr Neha Gupta, Consultant- Infectious Diseases, MEDANTA- The Medicity, Gurgaon, India, Tel+9l-8882927905, Email nehathdoc@gmail.com

Received: July 13, 2020 | Published: July 27, 2020

\section{Introduction}

Cryptococcosis is a fungal infection caused by Cryptococcus neoformans and Cryptococcus gattii and accounts for the most common cause of fungal meningitis worldwide. ${ }^{1}$ The range of clinical infections includes chronic skin infections, lung infections and meningoencephalitis. Delay in diagnosis of cryptococcal meningitis is associated with increased morbidity and mortality. ${ }^{2,3}$ Diagnostic modalities include cryptococcal antigen $(\mathrm{CrAg})$ detection tests, culture and the BioFire meningitis/encephalitis (ME) panel (bioMerieux, Marcy l'Etoile, France). Culture remains the gold standard for diagnosis of cryptococcal disease but it is time consuming and labor intensive. Cryptococcal antigen $(\mathrm{CrAg})$ lateral flow assays are rapid, specific, and may be more sensitive than culture when the burden of organism is low. ${ }^{4}$ However, in patients presenting with symptoms of meningoencephalitis, the causative organism is frequently not clinically apparent. There can be a large potential differential diagnosis requiring consideration, and there may be limited available CSF for testing. Efforts have been made in recent years to develop assays that would allow testing for a wide variety of pathogens associated with meningoencephalitis in a short time frame and using small volumes of CSF. The FilmArray Meningitis/Encephalitis (ME) Panel (BioFire Diagnostics, Salt Lake City, UT) uses multiplex polymerase chain reaction (PCR) to test for 14 most common bacterial, viral and fungal pathogens associated with Central Nervous System (CNS) infections in approximately one hour using just $0.2 \mathrm{ml}$ of CSF. ${ }^{5}$ The fast turnaround time allows clinicians to rapidly initiate targeted therapies. However, as with all diagnostic tools, clinical correlation is of paramount importance and clinicians need to be cautious in interpreting results from such PCR panels. Herein we report two cases of cryptococcal meningoencephalitis with false-negative results with the BioFire meningitis/encephalitis panel.

\section{Case I}

A 50-year gentleman sought medical attention in 2018 for headache of 5 days duration associated with weakness and fever with chills for the past 2-3 days. Headache was severe in intensity, diffuse, non-radiating and was not associated with nausea, vomiting, seizure, loss of consciousness or focal neurological deficit. He had undergone a live-donor kidney transplant 5years ago. He had subsequently developed chronic antibody mediated rejection (AMR) with a baseline creatinine $2.3 \mathrm{~g} / \mathrm{dl}$ and was receiving tacrolimus, azathioprine and prednisolone as immunosuppressive therapy. On examination, patient was conscious, well oriented to time, place, and person; vitals were stable, afebrile. On systemic examination, no abnormalities were detected. On neurological examination, neck rigidity was present; higher mental functions were normal with no cranial nerve abnormalities. Patient had no motor deficit and normal fundus examination. His laboratory evaluation showed hemoglobin (Hb) $9.1 \mathrm{gm} / \mathrm{dl}$, total leucocyte count (TLC) was $10590 \mathrm{cells} / \mathrm{cm}^{3}$ with platelets $1.93 \mathrm{lakh} /$ cumm, creatinine was $2.5 \mathrm{mg} / \mathrm{dl}$, and liver function tests were normal. Blood and urine cultures were sterile. Non-contrast computerized tomography (NCCT) brain revealed a few small focal hypodensities in supratentorial periventricular and subcortical white matter suggestive of chronic microvascular ischaemic changes. Rest of the brain parenchyma revealed normal attenuation. Magnetic resonance imaging (MRI) brain findings were suggestive of changes of Fazekas grade I to II chronic microvascular ischemic changes in supratentorial white matter. No acute infarct/intra-parenchymal or subdural hematoma/venous sinus thrombosis or intracranial spaceoccupying mass lesion was seen. No evidence of PRES/osmotic demyelination was seen. NCCT chest revealed few conglomerate nodular opacities seen in left upper lobe anterior segment with a possible infective etiology.

CSF analysis revealed total cell count (TCC) 284 cells $/ \mathrm{cm}^{3}(41 \%$ neutrophils, $58 \%$ lymphocytes), glucose of $78 \mathrm{mg} / \mathrm{dl}$, and protein of $362 \mathrm{~g} / \mathrm{dl}$, ADA- 3.90 and a negative Xpert MTB/RIF test. IndiaInk was negative; no fungal element seen on $\mathrm{KOH}$ mount; no acid fast bacilli seen on ZN staining; No pus cells and micro-organism seen on Gram staining; neither acid fast branching filamentous bacilli morphologically resembling Nocardia were seen on modified 
ZN staining. Cytomegalovirus (CMV) DNA was not detected. No organism was detected by rapid meningitis PCR (BioFire ME Panel) panel (included E.coli K1, H. influenzea, Listeria monocytogenes, Neisseria meningitides, streptococcus agalactiae, Streptococcus pneumoniae, Cytomegalovirus, Enterovirus, HSV1, HSV2, HHV6, Human parechovirus, Varicella zoster virus, Cryptococcus neoformans/gatti). But the cryptococcal antigen ( $\mathrm{CrAg}$ ) was detected in CSF by immune-chromatography test (lateral flow assay). CSF aerobic and anaerobic cultures were sterile. CSF fungal culture grew Cryptococcus neoformans after 5 days of incubation. Infectious diseases (ID) consultation was sought by the primary team and patient was initiated on induction therapy with liposomal amphotericin B (L-AmB) and flucytosine (5-FC).

\section{Case 2}

A 40-year old gentleman with well controlled Type 2 Diabetes Mellitus (T2DM) on oral hypoglycemic agent presented in January 2020 with fever, headache, multiple episodes of vomiting since 1 month and neck pain since last 2 days. Fever was intermittent. Headache was frontal, dull aching, non-radiating, mild to moderate in severity, increased when turning towards right and flexion of head. Patient is a resident of Delhi NCR and had a recent travel history to the urban areas in Uttar Pradesh and Gujarat. There was no history of skin rash or abscesses, injury or high-risk behavior. During illness, patient had consulted local doctors and was treated with antibiotics with no significant improvement. On examination, patient was conscious, well oriented to time, place and person; vitals were stable, afebrile. On systemic examination, no abnormalities were detected. On neurological examination, neck rigidity was present; higher mental functions were normal with no cranial nerve abnormalities. Patient had no motor deficit, power- $5 / 5$ in all limbs, extensor planter response, and normal fundus examination. Laboratory reports revealed TLC 11,100 cells $/ \mathrm{cm}^{3}$ with normal CRP, ESR ( $7 \mathrm{~mm} /$ hour), kidney function test, and $\mathrm{Hb}_{\mathrm{Alc}}-6.3 \%$. Contrast MRI brain screening revealed multifocal areas of signal alteration and enhancement in supratentorial brain parenchyma, brainstem and cerebellum. The imaging findings were reported to be suggestive of infective etiology with possibility non-specific viral encephalitis/cerebellitis (Figure 1). MRI spine contrast cervical revealed mild degenerative spondylotic changes; abnormal signal and enhancement in bilateral cerebellum; no significant spinal stenosis, cord compression; no abnormal leptomeningeal or intramedullary enhancement; no significant focal intramedullary lesion detected.

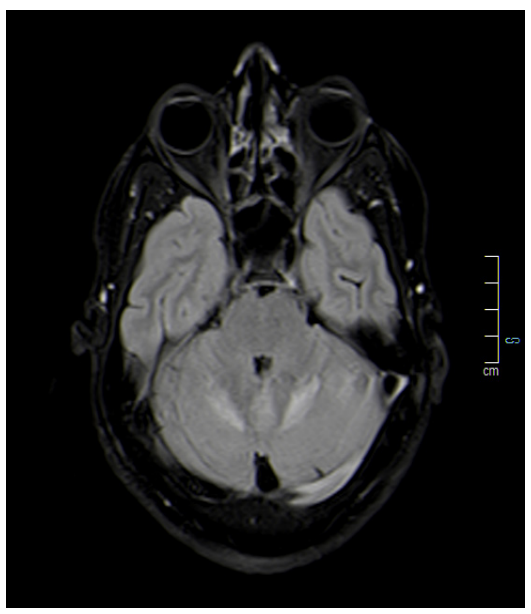

Figure I MRI brain suggestive of multifocal areas of signal alteration and enhancement in supratentorial brain parenchyma, brainstem and cerebellum.
Lumber puncture was performed and the CSF analysis revealed a TCC of $77 / \mu 1$ with $99 \%$ of lymphocytes, glucose- $88 \mathrm{mg} / \mathrm{dl}$, protein$70 \mathrm{mg} / \mathrm{dl}$; no organism seen on Grams stain; no fungal element seen on $\mathrm{KOH}$ mount; no acid fast bacilli seen on $\mathrm{ZN}$ stain; India Ink was negative for encapsulated budding yeast cells. Mycobacterium TB was not detected by Xpert MTB/RIF test; No organism was detected by Rapid meningitis PCR (film array) panel (included E.coli K1, H. influenzea, Listeria monocytogenes, Neisseria meningitides, streptococcus agalactiae, Streptococcus pneumoniae, Cytomegalovirus, Enterovirus, HSV1, HSV2, HHV6, Human parechovirus, Varicella zoster virus, Cryptococcus neoformans/gatti. CSF CrAg was positive by immunochromatography test (Figure 2). ID consultation was sought by the neurology team. Based on the history, imaging, and laboratory reports with a positive CrAg- a provisional diagnosis of cryptococcal meningoencephalitis was considered. Patient was started on L-AmB and 5-FC. The microbiology lab was requested to keep the CSF cultures for prolonged incubation of 15 days. Patient started improving on L-AmB and 5-FC. On $7^{\text {th }}$ day of aerobic culture, bottle flashed positive which showed budding yeast like cells on Gram stain (Figure 3) and grew on Sabouraud chloramphenicol agar (Figure 4). Identification and anti-fungal susceptibility was done by VITEK 2 Compact (bioMeriux) which showed Cryptococcal neoformans (sensitive to AmB, 5-FC, voriconazole, fluconazole) (Figure 5).

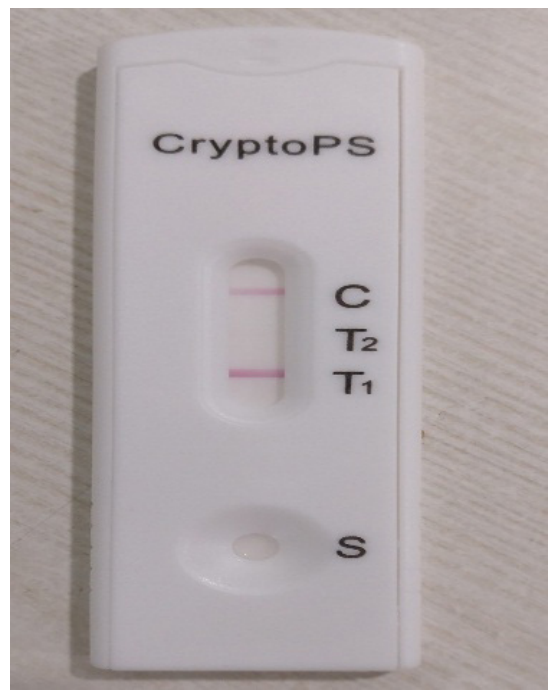

Figure 2 Cryptococcal Antigen ( $\mathrm{CrAg})$ was detected in CSF by immunochromatography test (lateral flow assay).

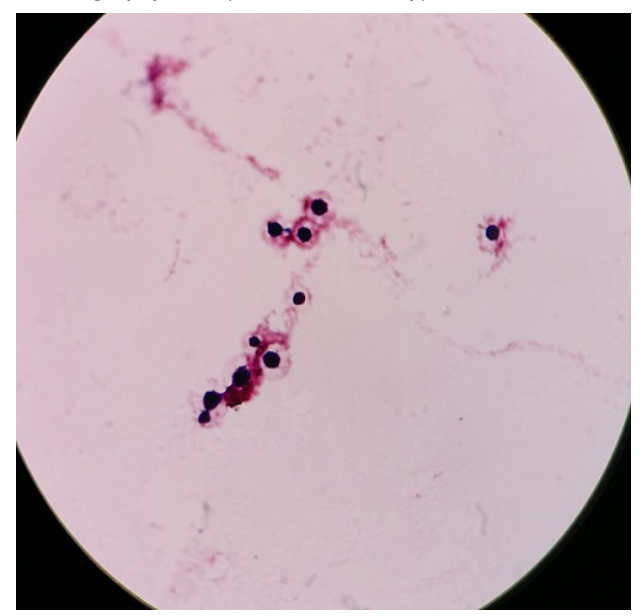

Figure 3 CSF containing bottle flashed positive which showed budding yeast cells on Gram stain. 


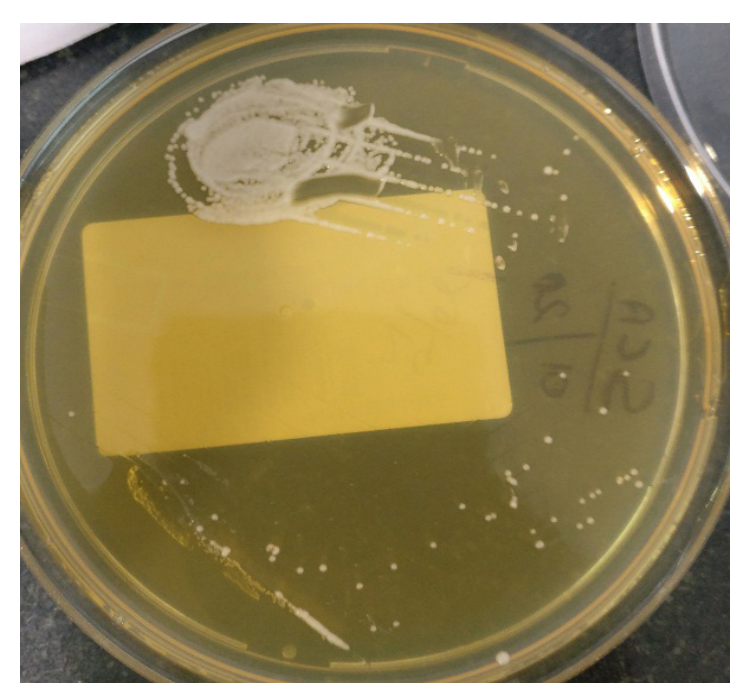

Figure 4 Growth of creamy white colonies of Cryptococcus on SCA media.

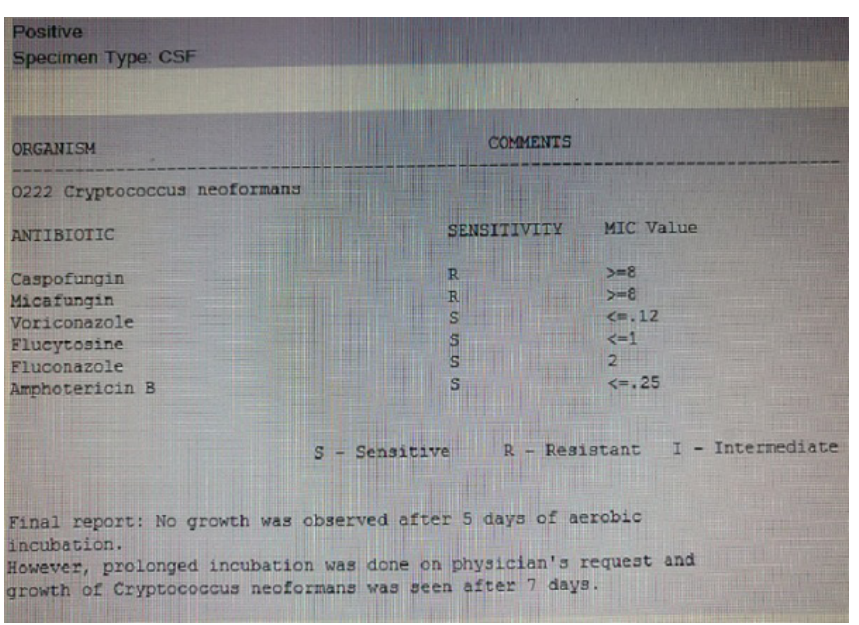

Figure 5 Identification and anti-fungal susceptibility report after prolonged incubation of CSF.

\section{Discussion}

These two cases highlight important pitfalls associated with the use of PCR panels such as the FilmArray ME Panel in evaluation of patients with meningoencephalitis important pitfalls. We describe one non-human immunodeficiency virus infected, non-transplant patient and one renal transplant recipient with cryptococcal meningitis with positive $\mathrm{CrAg}$ and Cryptococcus culture that was not detected on the BioFire ME panel. Fungal cultures were performed on brain heartinfusion agar $\left(37^{\circ} \mathrm{C}\right)$ and Sabouraud dextrose agar $\left(30^{\circ} \mathrm{C}\right)$ incubated in ambient air. Identification was initially performed by VITEK 2Compact (bioMerieux, Marcy l'Etoile, France). The reason for the false-negative results was thought to be due to low fungal counts below the lower limit of detection of the BioFire ME panel. According to the kit insert, the limit of detection of the assay was $100 \mathrm{CFU} / \mathrm{mL}$ for both Cryptococcus spp. On literature review, we found that the clinical assay performance of the FilmArray ME Panel was assessed in a multicenter study that examined 1560 residual CSF samples from patients undergoing lumbar puncture at 11 sites across the United States. In this study, the results from the FilmArray ME Panel were compared with comparator. The comparator was CSF culture for the bacteria and bidirectional PCR sequencing for the viruses and fungi included in the panel. The sensitivity for Cryptococcus neoformans/ gattii was reported at $100 \%(1 / 1)$ and was included in the overall assay sensitivity despite being calculated from a single positive specimen. Specificity for Cryptococcus spp. was reported as $99.7 \%(1555 / 1559)$. Of note, the comparator method for Cryptococcus in this study was PCR and not culture or CrAg testing. The investigators reviewed the records of the 4 patients who had positive results by the FilmArray ME Panel but negative results by the comparator PCR and determined that 2 were falsely negative by the comparator, and thus true positives detected by the FilmArray ME Panel. ${ }^{6}$

False-negative results of the FilmArray ME panel owing to low burden of the disease has been demonstrated in a smaller preclinical study. ${ }^{7}$ When the 16-target assay was performed on 342 stored CSF specimens, 8 of 14 (57\%) CrAg positive specimens were positive by the FilmArray ME panel, with 1 specimen that was CrAg negative/ FilmArray positive also testing positive by sequencing. The falsenegative results came from specimens with relatively low $\mathrm{CrAg}$ titers and/ or high PCR crossing thresholds, and therefore were likely related to low burden of disease. This may also explain why the FilmArray ME Panel performed well in subjects with first presentations of cryptococcal meningitis in a study performed in sub-Saharan Africa where the median quantitative culture was $8950 \mathrm{CFU} / \mathrm{mL}$ (IQR, 118-113 $500 \mathrm{CFU} / \mathrm{mL}$ ). Decreased sensitivity was observed in this study on follow-up CSF specimens obtained from therapeutic lumbar puncture in patients receiving appropriate antifungal therapy and presumably having lower fungal burden. In those with positive cryptococcal cultures and quantitative colony count $<100 \mathrm{CFU} /$ $\mathrm{mL}$, the sensitivity was $50 \%(6 / 12) .{ }^{8}, 9$ Another retrospective review identified five patients with cryptococcal meningoencephalitis, 4 of whom had a negative ME panel for Cryptococcus. All five cases had positive serum $\mathrm{CrAg}$, and three of five had a positive CSF culture for Cryptococcus. ${ }^{10}$ While the fungal cultures were positive on day 5 and day 7 respectively, time to culture positivity is dependent not only on fungal load but also on the volume of CSF inoculated, and hence, may not always represent burden of disease. On the other hand, the cryptococcal antigen LFA, which is performed with a fixed CSF volume, is more reproducible in quantifying fungal burden. The cryptococcal antigen LFA titre was positive in the undiluted sample for the both the patients. Although the BioFire ME panel may potentially yield a rapid diagnosis, clinicians should be cautious and aware that a negative cryptococcal result on this molecular platform does not rule out cryptococcal meningitis. Therefore, in clinical practice where there is a suspicion for cryptococcal meninigoencephalitis, assessment with multiple tests including CSF CrAg and culture should be performed to avoid delay in diagnosis.

\section{Acknowledgments}

The authors are thankful to Dr Naresh Trehan for his support.

\section{Conflicts of interest}

All Authors confirm that there is no conflict of interest.

\section{Funding source}

No funding support received for preparation of this manuscript.

\section{Ethical form}

The authors have obtained written and signed consent to publish the case report from the patients. 


\section{References}

1. Rajasingham R, Smith RM, Park BJ, et al. Global burden of disease of HIV associated cryptococcal meningitis: an updated analysis. Lancet Infect Dis. 2017;17:873-881.

2. Brizendine KD, Baddley JW, Pappas PG. Predictors of mortality and differences in clinical features among patients with Cryptococcosis according to immune status. PLoS One. 2013;8:e60431.

3. Aye C, Henderson A, Yu H, et al. Cryptococcosis-the impact of delay to diagnosis. Clin Microbiol Infect. 2016; 22:632-635.

4. Nalintya E, Kiggundu R, Meya D. Evolution of cryptococcal antigen testing: what is new? Curr Fungal Infect Rep. 2016;2016:1-6.

5. Wootton SH, Aguilera E, Salazar L, et al. Enhancing pathogen identification in patients with meningitis and a negative Gram stain using the BioFire FilmArray( $(\mathbb{B})$ Meningitis/Encephalitis panel. Ann Clin Microbiol Antimicrob. 2016; 15:26.
6. Leber AL, Everhart K, Balada-Llasat JM, et al. Multicenter evaluation of BioFire FilmArray Meningitis/Encephalitis panel for detection of bacteria, viruses, and yeast in cerebrospinal fluid specimens. $J$ Clin Microbiol. 2016;54:2251-2261.

7. Hanson KE, Slechta ES, Killpack JA, et al. Preclinical assessment of a fully automated multiplex PCR panel for detection of central nervous system pathogens. J Clin Microbiol. 2016 54:785-787.

8. Rhein J, Bahr NC, Hemmert AC, et al. ASTRO-CM Team. Diagnostic performance of a multiplex PCR assay for meningitis in an HIV-infected population in Uganda. Diagn Microbiol Infect Dis.2016;84:268-273.

9. O'Halloran JA, Franklin A, Lainhart W, et al. Pitfalls Associated With the Use of Molecular Diagnostic Panels in the Diagnosis of Cryptococcal Meningitis. Open Forum Infect Dis. 2017;4 (4):1-3.

10. Lewis PO, Lanier CG, Patel PD, et al. False negative diagnostic errors with polymerase chain reaction for the detection of cryptococcal meningoencephalitis. Med Mycol. 2020;58(3):408-410. 